\title{
Frequency-selective absorption characteristics of a metal surface with embedded dielectric microspheres
}

\author{
Gang Sun ${ }^{1,2}$ and C. T. Chan ${ }^{1}$ \\ ${ }^{1}$ Department of Physics, Hong Kong University of Science and Technology, Clear Water Bay, Kowloon, Hong Kong, China \\ ${ }^{2}$ Institute of Physics, Chinese Academy of Sciences, Beijing, China
}

(Received 12 January 2005; published 21 March 2006)

\begin{abstract}
The coupling of the resonances of spherical particles embedded near the surface of a metal and the surface plasmon is investigated by calculations using a multiple scattering formalism. The system we considered is a periodic array of glass spheres buried just beneath the surface of an Ag substrate. The calculated absorption spectra show features that can be traced to the resonance frequency of the microsphere cavity or the surface mode of the metal. In general, the absorption peaks for both the cavity and the surface mode are relatively weak when their resonance frequencies are far from each other. However, if the system is designed such that the cavitylike mode and the surface mode have almost the same frequency, the coupling of the resonances will lead to enhanced frequency-selective absorption. Such systems can be employed to modify or control thermal emission.
\end{abstract}

DOI: 10.1103/PhysRevE.73.036613

PACS number(s): 42.25.Bs, 73.20.Mf

A structured surface is useful in many important applications, and the majority of them, such as gratings, are employing the interference of the electromagnetic waves scattered by the surface structure. A structured surface can also yield some rather amazing phenomena through the excitation of resonances. For example, the recent experimental observation of enhanced optical transmission through metal films with periodic subwavelength holes, presumably enabled by the excitation of surface plasmons, has attracted considerable interest in the optical properties of such structures due to their numerous plausible applications in optics and optoelectronics as well as the rich physics behind the phenomenon of transmission enhancement [1-3]. Textured surfaces can also give "structural color" effects as observed in many animals in nature [4] and can be used to manipulate thermal radiation characteristics of emitting bodies [5]. The high transmittance of light through periodic arrays of subwavelength apertures has also received detailed theoretical analysis [6]. In this paper, we investigate a model system in which two resonances, namely the spatially localized cavitylike resonances of microspheres and the spatially extended surface plasmon of a metal, are coupled together and to the external light field via Bragg scattering. We will see that the coupling leads to strong resonances and absorption at some particular frequencies, depending on the size of the microspheres and how they are arranged. Since dielectric microspheres of various sizes (for example, silica spheres) are readily available, and the techniques to assemble and to control these spheres are increasingly sophisticated, such systems or their variants can be used to control the optical properties of a surface, leading to selective absorption or engineered thermal emission. We note that there has been some recent interest in employing photonic crystals [7] to engineer the thermal emission of materials. According to Kirchhoff's law, the emissive power is proportional to the absorbing power. A surface with frequency-selective absorbance will also give frequency-selective emittivity. Thus, the results of this paper are also relevant for the manipulation of the thermal radiation properties of surfaces. The coupled resonance system we consider may as well be a simpler alternative to photonic crystals as far as engineered thermal emission is concerned.

The model system we considered is a layer of dielectric (glass) microspheres embedded in a metal (Ag) substrate very close to the surface. The glass microspheres are arranged in a periodic triangular lattice, as sketched in Fig. 1. The nearest-neighbor distance between the spheres will be denoted as $\alpha$. The thickness of the cover layer $d_{c}$ (see Fig. 1 for definition) is chosen to be smaller than the skin depth so that external light can excite the resonances in the glass microspheres. In our calculations, the permittivity of the glass is set to be a constant with no absorption, $\varepsilon_{\mathrm{sph}}$ $=1.96$, and that of the silver $\varepsilon_{\mathrm{Ag}}$ is taken to be a Drude metal with plasma frequency $\omega_{p} \hbar=9.2 \mathrm{eV}$ and $\left(\omega_{p} \tau\right)^{-1}=0.02$, as generally used in the literature. The radius of the glass sphere is taken to be $S=0.1 \mu \mathrm{m}$. Other parameters, such as the nearest-neighbor distance between the spheres $(\alpha)$ and the thickness of the cover layer $d_{c}$, are varied so that we can observe the trend. The size of the spheres and the distance between them are chosen so that the frequencyselective absorption behaviors are observed in optical frequencies.

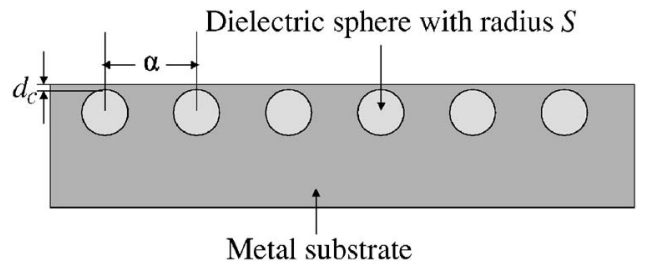

FIG. 1. A schematic sketch of the system we are investigating. Dielectric spheres of $\varepsilon=1.96$ and radius $0.1 \mu \mathrm{m}$ are embedded near the surface of an Ag host. The spheres are arranged in a triangular lattice with an intersphere distance of $\alpha$. The distance between the top of the spheres from the slab surface is given by $d_{c}$. 
One particular advantage of considering this simple system is that it is rich in physics, and yet it can be solved essentially exactly via a multiple scattering formalism. The cavitylike mode of a dielectric sphere embedded inside a metal can be solved analytically, and so is the dispersion of the surface plasmon. So, the basic resonances of the system have analytic solutions and they can be compared with the multiple scattering calculations to provide a clear picture of the coupling effect. More complex model systems, such as one with a corrugated Ag cover layer, would have qualitatively the same physics, but will make the interpretation of the results considerably more difficult.

Let us consider a single dielectric sphere embedded inside silver. There are some resonance frequencies, where some spherical harmonic electric modes can be excited if light can be coupled into the microsphere [8]. The resonance frequencies for each spherical harmonic can obtained from the peaks of coefficients $T_{l}^{E}(\omega)$ [9], which can be written as

$$
T_{l}^{E}(\omega)=\left.\frac{j_{l}\left(\kappa_{\mathrm{sph}} r\right) \frac{\partial}{\partial r}\left[r j_{l}\left(\kappa_{\mathrm{Ag}} r\right)\right] \varepsilon_{\mathrm{sph}}-j_{l}\left(\kappa_{\mathrm{Ag}} r\right) \frac{\partial}{\partial r}\left[r j_{l}\left(\kappa_{\mathrm{sph}} r\right)\right] \varepsilon_{\mathrm{Ag}}}{h_{l}^{+}\left(\kappa_{\mathrm{Ag}} r\right) \frac{\partial}{\partial r}\left[r j_{l}\left(\kappa_{\mathrm{sph}} r\right)\right] \varepsilon_{\mathrm{Ag}}-j_{l}\left(\kappa_{\mathrm{sph}} r\right) \frac{\partial}{\partial r}\left[r h_{l l}^{+}\left(\kappa_{\mathrm{Ag}} r\right)\right] \varepsilon_{\mathrm{sph}}}\right|_{r=S},
$$

where $c$ is the velocity of light in vacuum, and $j_{l}$ and $h_{l}$ are, respectively, spherical Bessel and Hankel functions, $\kappa_{\mathrm{sph}}$ $=\sqrt{\varepsilon_{\mathrm{sph}}} \omega / c$ and $\kappa_{\mathrm{Ag}}=\sqrt{\varepsilon_{\mathrm{Ag}}} \omega / c$. In this work, the radius of the glass sphere will be kept fixed throughout. Figure 2(a) shows the value of $T_{l}^{E}(\omega)$ for several spherical harmonic electric modes, labeled by the angular momentum index $l$. These coefficients give the ratio between the incident wave and the scattering wave for each angular momentum channel. A big value of the coefficient means a strong scattering, and if this coefficient diverges for some real values of $\omega$, it implies an eigenmode. We have an open and dissipative system in which the energy can couple in and out of the spherical cavity into the vacuum and into the surface mode, and energy can also be dissipated due to the imaginary part of the dielectric function. We cannot have true eigenmodes in the cavity, and thus a big value of the coefficient indicates a resonance, and the width of the resonance is related to the quality factor of the resonance. Figure 2(a) will be useful in identifying cavitylike resonances in the coupled systems, as we shall see in the next section. From Fig. 2(a), we see that the strongest resonance comes from the lowest frequency $l=1$ mode. The resonance becomes weaker and shifts to shorter wavelength as $l$ increases.

Now we consider the surface plasmon on the air-metal interface. For a flat metal-air interface, the surface plasmon has the dispersion relation

$$
k_{\mathrm{sp}}^{2}=\frac{\omega^{2}}{c^{2}}\left(\frac{\varepsilon_{\mathrm{Ag}}}{1+\varepsilon_{\mathrm{Ag}}}\right),
$$

where $k_{\mathrm{sp}}$ is the in-plane wave vector of the surface plasmon. The surface wave cannot be excited directly by an incident plane wave if the interface is perfectly flat, but it can be excited by evanescent waves, or by scattering centers near the surface. In particular, if there are periodic modulations on the surface, the Bragg scattering can fold the plasmon dispersion back into a free space light cone so that surface waves can be excited by incident plane waves. If there is a periodic surface modulation, the surface plasmon can couple with external propagating plane waves at the frequencies that satisfy

$$
\mathbf{k}_{\mathrm{sp}}=\mathbf{k}_{0} \sin \theta_{0} \pm p \mathbf{b}_{1} \pm q \mathbf{b}_{2},
$$

where $\mathbf{k}_{0}$ is the wave vector of the incident light in free space, $\theta_{0}$ is the angle of incidence, $\mathbf{b}_{1}$ and $\mathbf{b}_{2}$ are the reciprocal lattice vectors of the periodic surface, and $p$ and $q$ are integers. The surface plasmon can be viewed as an electromagnetic state confined near the air-metal interface, which can be excited by an external plane wave if the momentum conversation, as specified in Eq. (3), is satisfied. Among the surface waves that can be excited by an external plane wave, the strongest resonances (as measured by the absorption coefficient) are typically those with the lowest frequency, and the wave vector is equal to one of the reciprocal vectors of the lattice. For normal incidence, the lowest frequency surface plasmon that can be excited has a frequency given by

$$
\frac{2 \pi}{\alpha}=\frac{\sqrt{3}}{2} \frac{\omega}{c} \sqrt{\frac{\varepsilon_{\mathrm{Ag}}}{1+\varepsilon_{\mathrm{Ag}}}},
$$

where $\varepsilon_{\mathrm{Ag}}$ is the dielectric function of silver and $\alpha$ is the nearest-neighbor distance between two spheres in the triangular array.

While Eqs. (3) and (4) gives the kinematic conditions and the frequencies of allowed surface plasmon excitation, the coupling strength and the absorbance can only be determined by numerical computations. The multiple scattering method is particularly useful. The mathematical details of the method can be found in the literature [10-12]. This method expands the electromagnetic waves in terms of vector spherical harmonics centered on spherical scattering objects and is particularly suitable for systems that have spherical scatterers and flat interfaces; as a frequency-domain method, it can handle systems with dispersion and absorption as specified in the complex and frequency-dependent dielectric functions of the components. These are precisely what are needed in the 


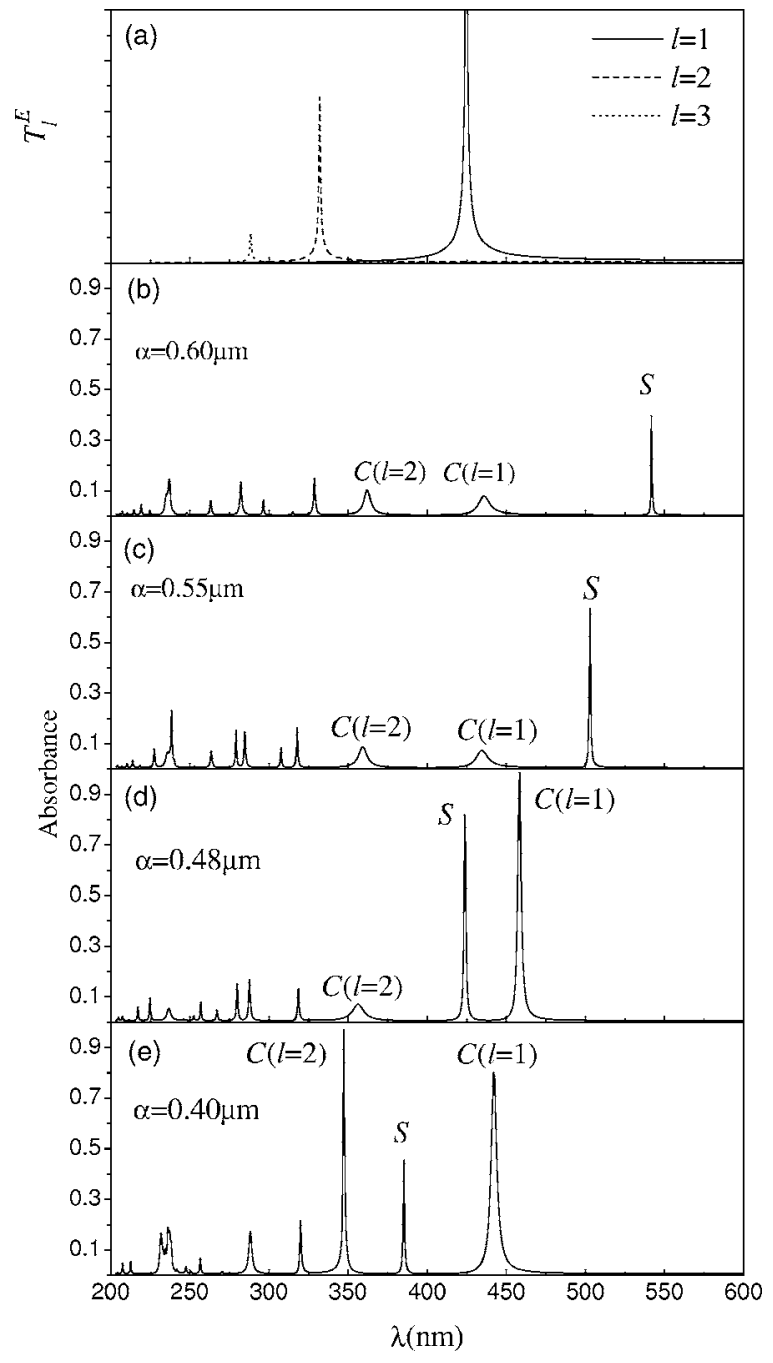

FIG. 2. (a) $T_{l}^{E}(\omega)$ (see text for definition) for several values of the angular momentum index $l$. The absorbance calculated for various lattice constants $\alpha$ from 0.6 to $0.4 \mu \mathrm{m}$ are shown in panels (b)-(e). The value of the cover layer thickness is fixed at $d_{c}=5 \mathrm{~nm}$. The symbols $S$ and $C$ label surface-plasmon-like and cavitylike resonances, respectively.

calculations. The convergence of the spherical wave expansion is controlled by the maximum value of the angular moment index, which will be called $L_{\max }$. The convergence criterion is found numerically by increasing the value of $L_{\max }$ until the results do not change. The convergence requirement for the system under investigation is rather demanding, especially when the spheres become close to each other. For this reason, we restrict our calculations to $\alpha>3 S$. By numerical convergence tests, we found that we need to use vector spherical harmonics with an angular momentum index as large as $L_{\max }=19$ to obtain reliable results for the most demanding case of the convergence, which corresponds to the smallest lattice constant and smallest thickness of the cover layer.

Figures 2(b)-2(e) show the results of the calculated absorbance as a function of the free-space wavelength $\left(\lambda=\frac{2 \pi c}{\omega}\right)$ for a series of intersphere distances. The cover layer thickness is fixed at $d_{c}=5 \mathrm{~nm}$. We will focus our attention on the lowest frequency absorption peaks, labeled by $S$ and $C$, respectively, in the figure. Figure 2(b) corresponds to the case in which the embedded glass spheres are $0.6 \mu \mathrm{m}$ apart from each other. In this panel, the absorption peak labeled $C(l=1)$ can be traced to the $l=1$ cavitylike mode of the embedded dielectric sphere. The fact that this is an $l=1$ mode can be identified by varying the maximum angular momentum index used in the multiple scattering calculations from one progressively to higher values. This $l=1$ cavitylike mode has nearly the same frequency as the cavitylike resonance frequency of a sphere embedded in bulk Ag, shown by the solid line in Fig. 2(a). The lower frequency peak labeled $S$ is due to the excitation of the surface plasmon on a periodically modulated metal-dielectric interface, which has frequency given approximately by Eq. (4). The Bragg scattering is enabled by the periodic array of spheres. When the lattice constant is relatively large, so that the microspheres are far away from each other $(\alpha=0.60 \mu \mathrm{m})$, the two resonance frequencies are far from each other, and both peaks are low and narrow [as shown in Fig. 2(b)], and their origins can be easily identified. Figure 2(c) shows the situation when the spheres are closer together, at $\alpha=0.55 \mu \mathrm{m}$. The cavitylike resonance remains at about the same frequency, as expected, while the plasmon peak moves to higher frequencies. When the distance between the spheres is progressively decreased, we see from panels (b) -(d) that the $l=1$ cavitylike mode remains more or less in the same position, while the plasmon peak increases in frequency and crosses the cavitylike mode. When the cavitylike resonance and the surface plasmon resonance are close in frequency, the coupling increases the absorbance of both resonances, as the absorption peaks become higher and wider.

We see from Fig. 2(d) that when the two resonance frequencies are about the same, at $\alpha=0.48 \mu \mathrm{m}$, both peaks reach the highest value. At that particular configuration, the assignment to cavitylike or plasmonlike resonance is somewhat difficult, and we will defer to the next paragraph to explain why we label the lowest frequency resonance as the cavitylike resonance, and the second lowest as the surfaceplasmon-like resonance. If we further decrease the lattice constant, the resonance frequency of the surface mode becomes higher than that of the cavity. At $\alpha=0.40 \mu \mathrm{m}$ [Fig. $2(\mathrm{e})]$, the surface-plasmon resonance is between $l=1$ and $l$ $=2$ cavity mode and the coupling leads to the enhancement of the absorption of both of the cavity resonances. We also note that the plasmon peak at $\alpha=0.48 \mu \mathrm{m}$ is actually stronger than at $\alpha=0.40 \mu \mathrm{m}$. From these figures, we see the synergistic effect of coupled resonances. At higher-frequencies, some small peaks can be traced to higher-order cavity resonances $(l=2$ and $l=3)$ and higher-order surface-plasmon modes. However, these modes are closely spaced in frequency and the coupling between the cavity mode and the surface-plasmon mode makes the assignment to plasmonlike or cavitylike resonances somewhat difficult. In Fig. 3, we consider the effect of the value of the parameter $\left(\omega_{p} \tau\right)^{-1}$ for the case of $\alpha=0.55 \mu \mathrm{m}$, in which the plasmon and the cavitylike resonances are clearly separated. A higher value of $\left(\omega_{p} \tau\right)^{-1}$ indicates higher absorption. The quality factor of the resonance, as reflected by the width of the absorption peak, is partly due to dissipation and partly due to coupling to 


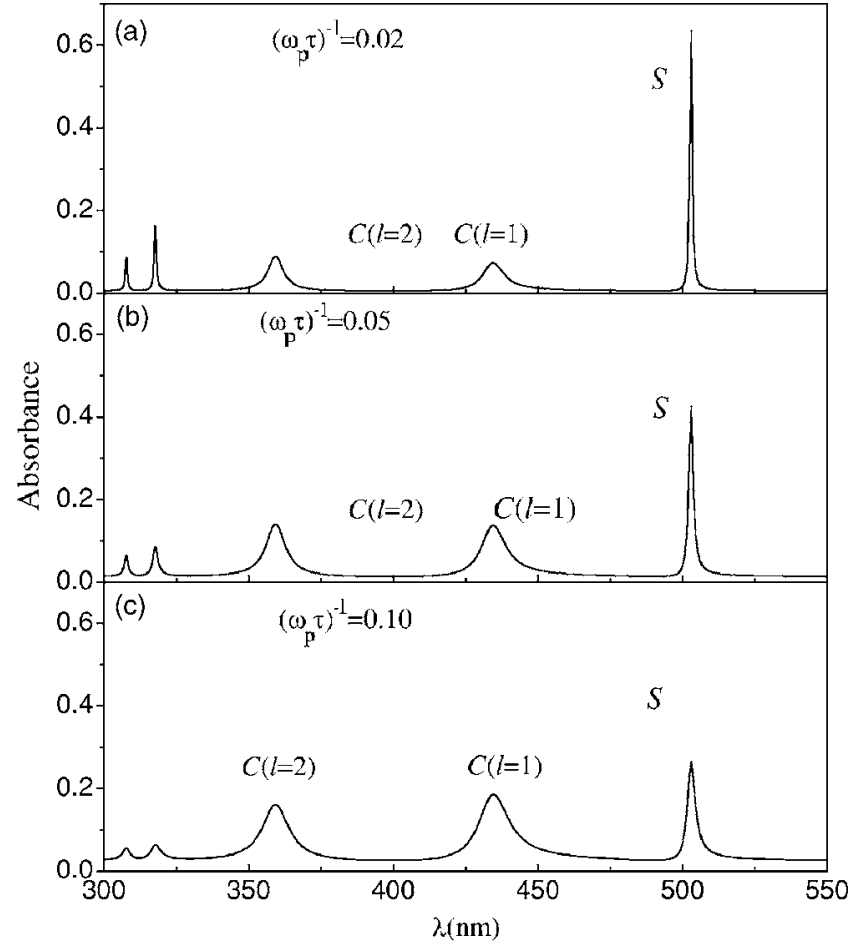

FIG. 3. The calculated absorbance for a fixed lattice constant $\alpha=0.55 \mu \mathrm{m}$ with various values of $\left(\omega_{p} \tau\right)^{-1}$. The $x$ axis is labeled by the free-space wavelength.

other modes such as the radiative modes in the vacuum. By varying $\left(\omega_{p} \tau\right)^{-1}$ from 0.02 to 0.10 , we see that the peak derived from the surface plasmon (labeled $S$ ) is affected more than the peaks due to the cavitylike resonance of the spheres (labeled $C$ ). For the cavity-derived peak, higher values $\left(\omega_{p} \tau\right)^{-1}$ lead to a higher and broader peak; both are natural consequences of the higher dissipation (and absorption) in a localized mode. The surface-plasmon peak becomes weaker as $\left(\omega_{p} \tau\right)^{-1}$ increases. The surface-plasmon excitation of a precise and specific value of the parallel momentum requires an infinite periodic array to excite. When the dissipation is increased, the coherence length of multiple scattering is limited in real space, and the decrease of coherence leads to a corresponding uncertainty in momentum and thus a weaker and broader peak.

Since the glass microspheres are embedded, the thickness of the cover layer of the metal affects the coupling between the incident plane wave and the cavity mode, as well as that between the cavity mode and surface-plasmon mode. Figure 4 shows the splitting of the resonance mode for various thicknesses of the cover layer at the lattice constant of $\alpha$ $=0.47 \mu \mathrm{m}$. The two dominant peaks are between 400 and $500 \mathrm{~nm}$. We note that the higher-frequency peak at about $420 \mathrm{~nm}$ stays essentially at the same frequency, while the lower-frequency resonance shifts to lower frequencies as the top layer distance $d_{c}$ decreases. We expect that the microsphere resonance frequency should depend more sensitively on the top layer distance, which is smaller than the skin depth, while the surface-plasmon frequency is a property of the silver surface, and will not be affected much. It is for this reason that we have labeled the lower-frequency resonance

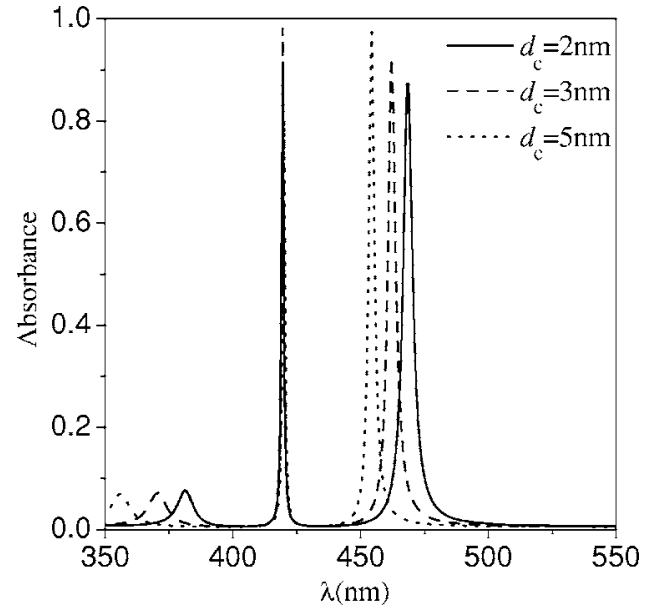

FIG. 4. The absorbance for a fixed lattice constant $\alpha=0.47 \mu \mathrm{m}$ with various thicknesses of the cover layer $d_{c}$. The $x$ axis is labeled by the free-space wavelength.

as the cavity resonance and the higher-frequency peak as the surface-plasmon-like resonance in Fig. 2(d). In Fig. 5, we show the wavelengths of the lowest and second-lowest absorption peaks as a function of $\alpha$ for various covering layer thickness $d_{c}$. The cavitylike mode $l=1$ resonance and the lowest frequency surface-plasmon resonance [as given by Eq. (4)] are plotted as dotted and solid lines, respectively, in Fig. 4. Far away from the crossing point of the dotted and solid line, the assignment of the resonance to plasmonlike or cavitylike is visually obvious. When the solid and dotted lines cross, the two resonances are of the same frequency, but the coupling splits them and the splitting is larger for a thinner cover layer. This is also evident in Fig. 4. We also see that the position of the resonance peak corresponding to the cavity mode depends more sensitively on the thickness of the cover layer. This is quite understandable since the cavitylike

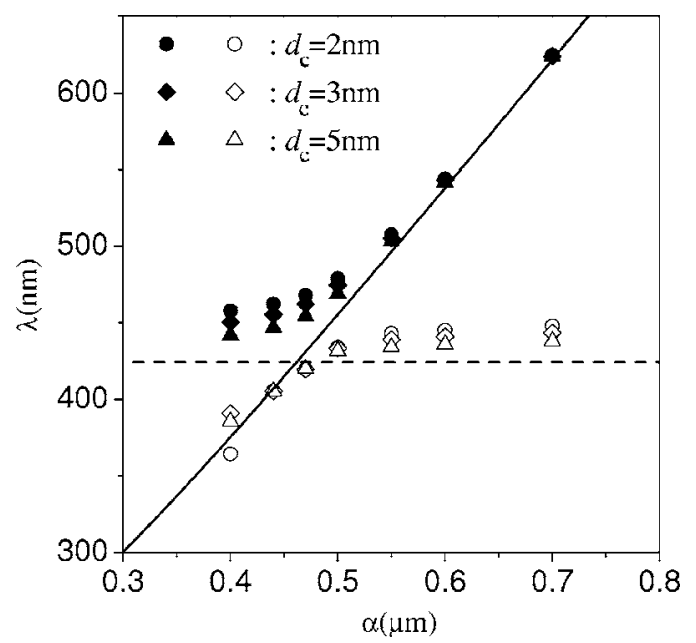

FIG. 5. The free-space wavelengths of the peaks at the lowest and second-lowest frequencies for several thicknesses of the cover layer are plotted as a function of the interparticle distance $\alpha$. As a reference, the wavelength of the surface-plasmon resonance is shown as a solid line and that for the cavity (dielectric sphere embedded in bulk $\mathrm{Ag}$ ) is marked by a dashed line. 
resonance frequency depends on the boundary condition, and in this case, it depends on the leakage of the mode into the vacuum. In Fig. 5, the dotted line marks the $l=1$ resonance for a glass sphere inside a bulk Ag. The numerically calculated cavity mode resonance frequency here deviates from that value since it is covered by a thin cap on the top, rather than surrounded by bulk $\mathrm{Ag}$ on all sides. The surfaceplasmon peaks approach the analytic value at larger separations of the spheres.

If we take a look at Figs. 2(d) and 2(e) again, we see that the coupling of the resonances leads to very high absorption at some particular frequencies. These systems will thus have structural color, and they will have frequency-selective absorption and emissivity. The thermal emissivity of these surfaces will have selective emissive bands and deviate strongly from that of a black or white body. There is also another way that we can view Fig. 5 if we focus on one particular value of the top layer thickness, say at $d_{c}=5 \mathrm{~nm}$. Since $\lambda \sim 1 / \omega$ and $\alpha \sim 1 / k$, Fig. 5 can be viewed as a dispersion diagram for the surface mode, and due to the coupling with the localized resonance of the microsphere, a surface wave spectral gap is opened at the cavity resonance frequency of the embedded microsphere. This surface wave band gap is somewhat different from the usual photonic band gap, which is derived from Bragg scattering, in which the gap frequency is determined by the lattice constant. Here, the surface wave gap is derived from localized resonances, with its gap frequency pinned at the cavity resonance frequency, and the role of Bragg scattering is to provide a mechanism to couple to the surface waves.

We have been focusing on the case of normal incidence. When light is incident at an angle, we find that the resonance peak due to the cavity mode is almost unchanged. The surface-plasmon peak position can be predicted by Eq. (3) as long as the frequency is far away from the microsphere modes. At normal incidence, a set of reciprocal vectors having the same length will satisfy the same kinematics condi- tions, leading to one single absorption peak. For example, in the triangular lattice we have considered, the set of six symmetry-related reciprocal vectors $\pm \mathbf{b}_{1}, \pm \mathbf{b}_{2}, \pm\left(\mathbf{b}_{1}-\mathbf{b}_{2}\right)$ fold the plasmon band back to the same point at the zone center, leading to one single plasmon absorption peak. However, if incidence is not normal, then each one of these reciprocal lattice vectors can couple the external light to a different $\mathbf{k}_{\mathrm{sp}}$, leading to a multiple of absorption peaks. So, instead of the single dominant plasmon peak, such as the one labeled $S$ in Fig. 2, the surface-plasmon resonance would split into a few peaks, and the amplitude of these split peaks will depend on polarization of the E-field. The situation is thus rather complex. However, when the cavity mode and the plasmon modes are close together, the coupling will lead to mutual enhancement, as in the case of normal incidence.

In summary, we used the multiple scattering method to calculate and understand the absorption characteristics of a metal surface with an array of embedded microspheres. This model system has the advantage that the numerical calculations can be carried to a high precision by the multiple scattering method, and the physics can be traced and understood to great detail. The coupling of the localized cavitylike resonances and extended surface wave resonances leads to strong frequency-selective absorption, and these configurations are also plausible candidates for controlled thermal emission surfaces. Lastly, we note that the focus of this paper is on the theoretical analysis of a model system, which is chosen for the convenience of theoretical treatment. However, with the rapid advance in nanoscience techniques [13] and in the fabrication and ordering of metal-coated spheres, systems with configurations similar to those described in the paper may be realizable in laboratories. In fact, subsequent to the submission of this paper, a system similar to that modeled by us was made using electrochemical methods [14].

This work is supported by Hong Kong RGC through Grant No. 600403.
[1] T. W. Ebbesen, H. J. Lezec, H. F. Ghaemi, T. Thio, and P. A. Wolff, Nature (London) 391, 667 (1998).

[2] H. F. Ghaemi, T. Thio, D. E. Grupp, T. W. Ebbesen, and H. J. Lezec, Phys. Rev. B 58, 6779 (1998).

[3] U. Schröter and D. Heitmann, Phys. Rev. B 58, 15419 (1998).

[4] See, e. g., P. Vukusic and J. R. Sambles, Nature (London) 424, 852 (2003).

[5] S. Maruyama, T. Kashiwa, H. Yugami, and M. Esashi, Appl. Phys. Lett. 79, 1393 (2001); H. Sai, H. Yugami, Y. Akiyama, Y. Kanamori, and K. Hane, J. Opt. Soc. Am. A 18, 1471 (2001)

[6] See, e.g., J. A. Porto, F. J. Garcia-Vidal, and J. B. Pendry, Phys. Rev. Lett. 83, 2845 (1999); L. Martin-Moreno, F. J. Garcia-Vidal, H. J. Lezec, K. M. Pellerin, T. Thio, J. B. Pendry, and T. W. Ebbesen, ibid. 86, 1114 (2001).

[7] See, e. g., J. G. Fleming, S. Y. Lin, I. El-Kady, R. Biswas, and K. M. Ho, Nature (London) 417, 52 (2002).

[8] See, e.g., C. F. Bohren and D. R. Huffman, Absorption and
Scattering of Light by Small Particles (Wiley, New York, 1983).

[9] A. Modinos, Physica A 141, 575 (1987).

[10] V. Yannopapas, A. Modinos, and N. Stefanou, Phys. Rev. B 60, 5359 (1999).

[11] N. Stefanou, V. Yannopapas, and A. Modinos, Comput. Phys. Commun. 113, 49 (1998); N. Stefanou, V. Yannopapas, and A. Modinos, ibid. 132, 189 (2000).

[12] We note that the Ewald sum used in the computation of the " $Z$ " matrices is replaced by direct summation.

[13] See, e.g., E. Prodan, C. Radloff, H. J. Halas, and P. Nordlander, Science 302, 419 (2003); S. J. Oldenburg, R. D. Averitt, S. L. Westcott, and N. J. Halas, Chem. Phys. Lett. 288, 243 (1998); C. Graf and A. van Blaaderen, Langmuir 18, 524 (2002); S. L. Westcott, J. B. Jackson, C. Radloff, and N. J. Halas, Phys. Rev. B 66, 155431 (2002).

[14] T. A. Kelf, Y. Sugawara, J. J. Baumberg, M. Abdelsalam, and P. N. Bartlett, Phys. Rev. Lett. 95, 116802 (2005). 